\title{
An Innovative Method for Endovascular Stabilization of Vulnerable Plaque in Coronary Arteries: An Opinion
}

\author{
Alexander G Viller* and Viacheslav M Solomannikov \\ Innovative Endovascular Technologies LLC, Skolkovo Foundation, Moscow, Russia
}

*Corresponding author: Alexander G. Viller, Innovative Endovascular Technologies LLC, Skolkovo Foundation, Moscow, Russia

\section{Short Communication}

Despite all of the available diagnostic and treatment modalities atherosclerosis remains one of the most common healthcare problems worldwide with an estimated annual mortality rate of approximately 17,5 million cases [1]. In most cases acute coronary syndrome (ACS) appears to be linked to atherosclerotic lesion associated thrombosis of a vessel [2]. In the situation, when patients come to the interventional cardiology unit with a confirmed diagnosis of ACS: myocardial infarction, a so-called culprit lesion can be identified and treated according to international guidelines. However, alongside the lesions, that are obviously causing an impairment of a blood perfusion in a certain segment of the myocardium or have already destabilized causing acute thrombosis, some other form of entity can frequently be seen. These are called non - culprit lesions. Even though their appearance in an orifice of a vessel is regarded by most surgeons as a "bad omen", the international community is still puzzled and unsure, if these lesions are to be treated. And even if they are, the specialists cannot yet be sure, what type of an intervention is preferable. This problem arises from several premises, including the following:

I. The non - culprit vulnerable plaque destabilization is a complex phenomenon. Not only the mechanical properties and structural integrity of the plaque define the further events, but the mechanical forces [3-5], that affect the plaque and are being transduces by blood flow, a non-Newtonian fluid, whose properties might be affected in a large variety of conditions. Apparently, the hemostasis must be accessed prior to drawing the right decision in patient's follow - up tactics. But exact parameters are yet to be determined [6].

II. The non - culprit vulnerable plaque destabilization does not always cause an ACS [7]. Some specialists imply that assessment of blood properties and structural characteristics of the plaque are not enough. Apparently, the state of a myocardium must also be assessed.
III. Not all vulnerable plaques are equal, and the risks of destabilization accompanied by MACE vary depending on the plaque localization [8]. This statement appears to be obvious. Most surgeons would have guessed this without any researches: the more approximate position in a coronary artery clearly indicates a higher chance of an unfavorable outcome. However, what might be not so apparent, the different segments of coronary arteries' do not react to sheer-stress in a uniform manner [9]. This is almost impossible to assess in real clinical practice but is a good thing to bear in mind.

One must put a lot of effort into the diagnostic procedures, in order to understand the whole situation. Simultaneously we do not know what kind of a medication must be used in every situation. A lot of effort has been put recently into finding a preferable drug for stabilization of a vulnerable plaque. Different approaches were used. Considering the trails REVERSAL, SATURN, ASTEROID and most importantly YELLOW, the conventional statins are still a medication of choice for the most patients with vulnerable plaques. Other drugs were introduced recently, including ivabradine [10], grelin [11], canakinumab [12]. Most of these approaches are still in early development, but it is clear now, that the lack of preventive conservative treatment is a risk factor for any manipulation [13], that is to be performed upon a vulnerable plaque.

Some cardiologists imply that more aggressive invasive techniques should be prioritized. We possess only scarce data. PRAMI trial indicates, that stenting any lesion, that is even less than 50\% might be beneficial [14]. The CvLPRIP trial has mostly confirmed the findings of PRAMI [15]. Interesting results were obtained by Dai et al [16] they were able to demonstrate, that routine stenting of all lesions leads to lower rates of death, secondary ACS. MACCE were lower: RR (HR) 0.35 [95\% CI 0.18 - 0.69]. Additional factors listed above might contribute to drawing a right decision in different situations. 
The problem with invasive procedures extends beyond that:

I. The risks of stent - thrombosis are unacceptable in endovascular plaque stabilization. This in turn requires at least using an extremely precise tool, that has only limited contact with endothelial cells, that have not yet succumbed to pathological process. Extensive damage frequently leads to neointimal hyperplasia, neoatherosclerosis and stent thrombosis. Considering all things stated, we suppose, that only truncated stents might be used for vulnerable plaque stabilization.

II. The usage of truncated stents requires a fairly good level of precision, that most devices nowadays are still unable to provide.

III. The risks of bleeding are also high, because a person is forced to take a variety of anticoagulants for a prolonged period after the procedure. The bigger the time interval, the bigger the chances of a major bleeding. Therefore, there is a question to be asked: what if the biodegradable stents, that did not legitimately receive much attention due to the known issues and inability to compete with DES in terms of conventional stenting, can be used in this situation.
IV. The risks of periprocedural myocardial infarction [17] are dependent on structural properties of the plaque. High lipid burden and large lipid core are mostly responsible for this adverse event [18]. This cannot be manipulated by the means of surgical instruments and is a single reason for carefully assessing the situation using different diagnostic techniques.

V. It is economically ineffective to create a separate device for treatment of vulnerable plaques only.

VI. Bearing in mind all the concerns stated, we decided to come up with a new device that can be used in many different fields but is also capable to satisfy all the precautions involved in vulnerable plaque endovascular stabilization. The throughout description of the basics of this over the wire stent delivery systems (SDS) construction is not however the goal of this article. All the information can be found in our patent here: US 20100070014 A1 published in 2010. The project is in early development and we do not encourage a reader to buy it. Therefore, this article is not a commercial, but a call to international specialists, that would probably find this topic interesting to discuss. The basic structure of the SDSs distal shaft is shown below (Figure 1).

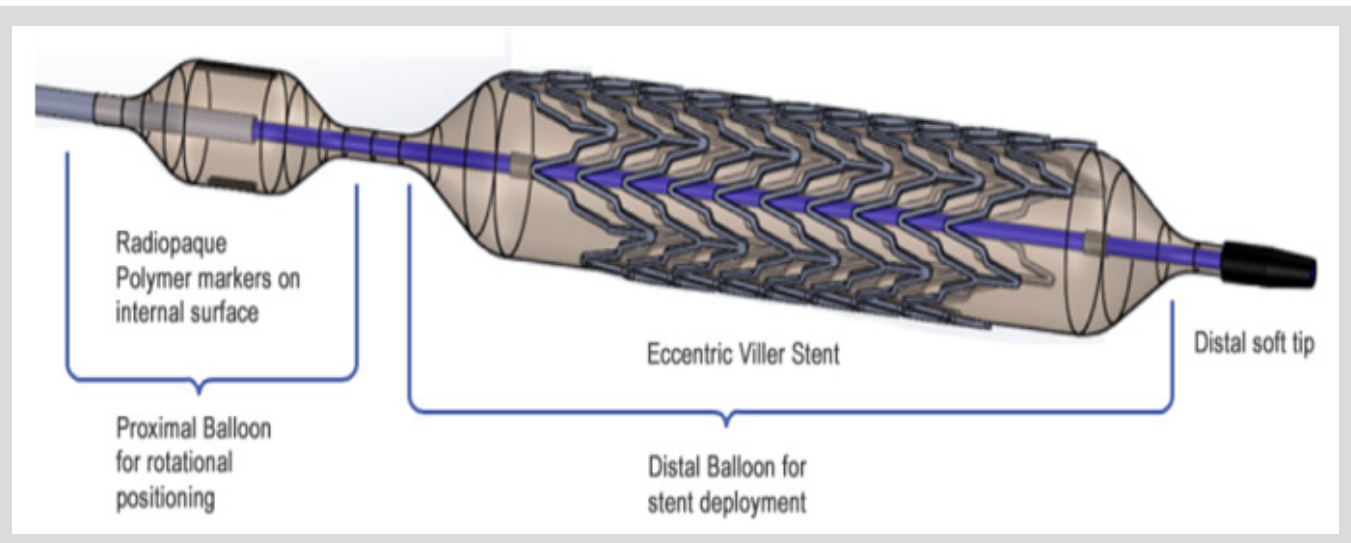

Figure 1.

Such SDS works in following order. Both balloons are connected consecutively to the compressor, but radiopaque label bearing balloon is more compliant and expands in the first place allowing the precise positioning of asymmetrical truncated stent in the orifice of the vessel. By applying sufficient force, the operator can ensure that the stent is in position, meanwhile, by applying additional pressure, can start the expansion of the second stent- bearing balloon. After the implantation is complete the SDS is removed at once. We are currently developing new biodegradable stents and will test the whole system on a swine model in several months.

\section{References}

1 Stefanadis C, Antoniou C, Tsiachris D, Pietri P (2017) Coronary Atherosclerotic Vulnerable Plaque: Current Perspectives. J Am Heart Assoc 6(3): e005543.

2 Naghavi M, Libby P, Falk E, Casscells SW, Litovsky S, et al. (2003) From Vulnerable Plaque to Vulnerable Patient: A Call for New Definitions and Risk Assessment Strategies: Part II. Circulation 108(15): 1772-1778.

3 Virmani R, Kolodgie FD, Burke AP, Farb A, Schwartz SM (2000) Lessons from sudden coronary death: a comprehensive morphological classification scheme for atherosclerotic lesions. Arterioscler Thromb Vasc Biol 20(5): 1262-1275.

4 Ohayon J, Finet G, Gharib AM, Herzka DA, Tracqui P, et al. (2008) Necrotic core thickness and positive arterial remodeling index: emergent biomechanical factors for evaluating the risk of plaque rupture. Am J Physiol Heart Circ Physiol 295(2): H717-27.

5 Krishnan S, Otaki Y, Doris M, Slipczuk L, Arnson Y, et al. (2017) Molecular Imaging of Vulnerable Coronary Plaque: A Pathophysiologic Perspective. J Nucl Med 58(3) : 359-364.

6 Yuhua S, Baoping W (2018) From vulnerable plaque to blood healthy therapy. Perfusion 33(2): 89-95.

7 Arbustini E, Grasso M, Diegoli M, Pucci A, Bramerio M, et al. (1991) Coronary atherosclerotic plaques with and without thrombus in ischemic heart syndromes: a morphologic, immunohistochemical, and biochemical study. Am J Cardiol 68(7): 36B-50B.

8 Wang JC, Normand SLT, Mauri L, Kuntz RE (2004) Coronary Artery Spatial Distribution of Acute Myocardial Infarction Occlusions. Circulation 110(3): 278-284.

9 Waxman S, Ishibashi F, Muller JE (2006) Detection and Treatment of Vulnerable Plaques and Vulnerable Patients: Novel Approaches to Prevention of Coronary Events. Circulation 114(22): 2390-411. 
10 van Hoof RHM, Hermeling E, Sluimer IC, Salzmann J, Hoeks APG, et al. (20147) Heart rate lowering treatment leads to a reduction in vulnerable plaque features in atherosclerotic rabbits. PLoS One 12(6): e0179024.

11 Wang L, Chen Q, Ke D, Li G (2017) Ghrelin inhibits atherosclerotic plaque angiogenesis and promotes plaque stability in a rabbit atherosclerotic model. Peptides 90: 17-26.

12 Ridker PM, Everett BM, Thuren T, MacFadyen JG, Chang WH, et al. (2017) Antiinflammatory Therapy with Canakinumab for Atherosclerotic Disease. N Engl J Med 377(12): 1119-1131.

13 Hougaard M, Hansen HS, Thayssen P, Antonsen L, Jensen LO (2018) Uncovered Culprit Plaque Ruptures in Patients With ST-Segment Elevation Myocardial Infarction Assessed by Optical Coherence Tomography and Intravascular Ultrasound With iMap. JACC Cardiovasc Imaging 11(6): 859-867.

14 Wald DS, Morris JK, Wald NJ, Chase AJ, Edwards RJ, et al. (2013) Randomized Trial of Preventive Angioplasty in Myocardial Infarction. N Engl J Med 369(12): 1115-1123.
15 Kelly DJ, McCann GP, Blackman D, Curzen NP, Dalby M, et al. (2013) Complete Versus Culprit-Lesion only PRimary PCI Trial (CVLPRIT): a multicentre trial testing management strategies when multivessel disease is detected at the time of primary PCI: rationale and design. EuroIntervention 8(10): 1190-1198.

16 Dai J, Lyu S, Song X, Zhang M, Nie S, et al. (2017) Stent Versus Non-Stent in Treating Intermediate Stenosis Culprit Lesions in Acute ST-Segment Elevation Myocardial Infarction Patients. Int Heart J 58(3) : 357-364.

17 Kini AS, Motoyama S, Vengrenyuk Y, Feig JE, Pena J, et al. (2015) Multimodality Intravascular Imaging to Predict Periprocedural Myocardial Infarction During Percutaneous Coronary Intervention. JACC Cardiovasc Interv 8(7) : 937-945.

18 Kini AS, Baber U, Kovacic JC, Limaye A, Ali ZA, et al. (2013) Changes in plaque lipid content after short-term intensive versus standard statin therapy: the YELLOW trial (reduction in yellow plaque by aggressive lipid-lowering therapy). J Am Coll Cardiol 62(1) : 21-29.

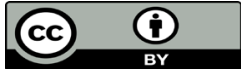

This work is licensed under Creative Commons Attribution 4.0 License

To Submit Your Article Click Here:

Submit Article

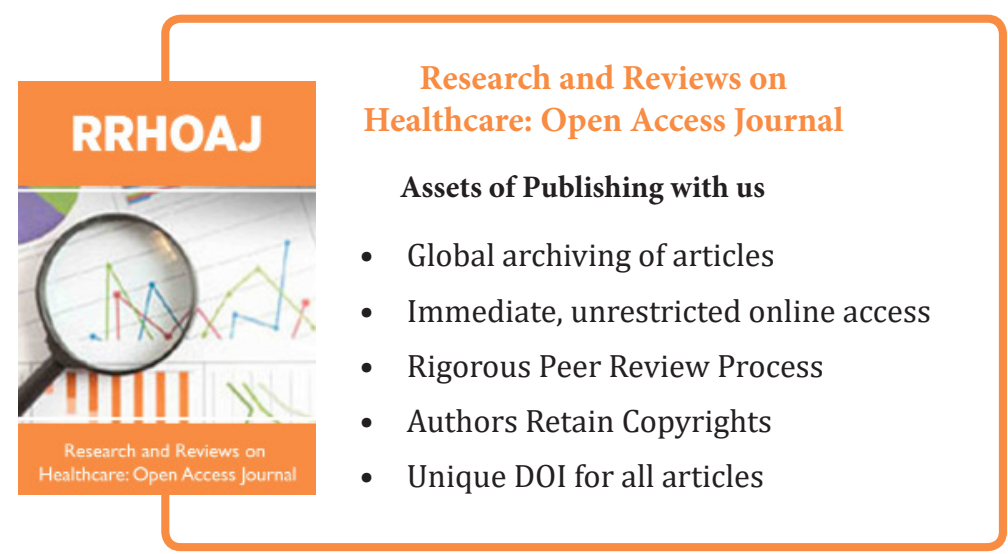

Citation: Alexander G Viller, Viacheslav M Solomannikov. An Innovative Method for Endovascular Stabilization of Vulnerable Plaque in 